\title{
Mammographic Findings Associated with Accelerated Partial Breast Irradiation Using Single Fraction Intraoperative Radiotherapy
}

\author{
Kathleen C. Horst ${ }^{1}$, Debra M. Ikeda ${ }^{2}$, Katherine E. Fero ${ }^{1}$, Jafi A. Lipson ${ }^{2}$, Sunita Pal ${ }^{2}$, \\ Don R. Goffinet ${ }^{1}$, Frederick M. Dirbas ${ }^{3}$ \\ ${ }^{1}$ Departments of Radiation Oncology, Stanford University, Stanford, USA; ${ }^{2}$ Departments of Diagnostic Radiology, Stanford Univer- \\ sity, Stanford, USA; ${ }^{3}$ Departments of Surgery, Stanford University, Stanford, USA. \\ Email:kateh@stanford.edu
}

Received June $15^{\text {th }}, 2012$; revised July $18^{\text {th }}, 2012$; accepted July $29^{\text {th }}, 2012$

\begin{abstract}
Purpose: To evaluate the mammographic findings of women treated with accelerated partial breast irradiation (APBI) using single-fraction intraoperative radiotherapy (IORT). Materials/Methods: Women $\geq 40$ years of age with unifocal invasive or intraductal carcinoma $\leq 2.5 \mathrm{~cm}$ on physical examination, mammography, and ultrasound were enrolled on an APBI trial using single fraction IORT. Post-treatment mammographic imaging was obtained at 6 months, 1 year, and then annually. Results: Between 12/02 and 6/04, 17 women underwent IORT at the time of lumpectomy (median age $=$ 60 years; range $=40-83$ ). The initial post-IORT mammogram showed increased density at the lumpectomy site in 11 patients $(65 \%)$, while six patients $(35 \%)$ had architectural distortion in the area of the irradiated tissue. Fifteen patients $(88 \%)$ had numerous punctate, benign-appearing calcifications corresponding to the irradiated region. There was focal skin thickening near the incision in 13 patients (76\%). At a median of 67 months, architectural distortion had stabilized and the benign-appearing calcifications remained stable in number and character. Eight patients (47\%) had mammographic findings consistent with fat necrosis, ranging in size from $0.5-4 \mathrm{~cm}$. Conclusions: After lumpectomy and IORT, mammographic changes include increased density and benign appearing calcifications in the irradiated region with focal skin thickening. These changes appear to stabilize over time and are consistent with post-treatment changes. These changes are important to identify in order to characterize benign changes from recurrent tumor.
\end{abstract}

Keywords: Accelerated Partial Breast Irradiation (APBI); Intraoperative Radiotherapy (IORT); Breast Cancer; Mammography; Microcalcifications; Fat Necrosis

\section{Introduction}

Multiple prospective, randomized trials have demonstrated that whole-breast irradiation (WBI) reduces the risk of ipsilateral breast tumor recurrence (IBTR) after breast conserving surgery (BCS) [1-6]. Given these longterm results, the combination of BCS and WBI has been considered a standard treatment option for women with early stage breast cancer [7].

More recently, accelerated partial breast irradiation (APBI) has emerged as an alternative to WBI [8]. APBI targets the lumpectomy cavity plus a margin of normal breast tissue and allows for treatment to be delivered in 1 - 5 days compared to the standard 6 - 7 weeks with WBI. Phase III trials comparing WBI versus APBI are ongoing in Canada, Europe, and the United States. Various techniques are being utilized, including interstitial or intracavitary brachytherapy, 3D conformal radiotherapy
(3D-CRT), and single fraction intraoperative radiotherapy (IORT) [9-12]. A recently published phase III trial evaluating IORT using the Intrabeam $^{\circledR}$ device demonstrated equivalent recurrence rates and toxicity compared to WBI [13].

The increasing use of APBI raises questions regarding the early and long-term safety of the higher radiation doses per fraction [14]. In addition to toxicity, there are concerns about alterations in mammographic findings that may hinder the ability to detect recurrences $[15,16]$. It is therefore important to characterize mammographic changes after APBI in order to limit fals positive findings that could lead to unnecessary biopsies.

At our institution, single-fraction IORT has been offered to women with early stage breast cancer. Data from European centers has suggested the approach is safe and effective $[13,17]$. However, the single, large radiotherapy dose delivered with IORT has raised concerns for some 
that it may be associated with even greater sequelae compared with other forms of APBI [18]. The aim of our study was to describe the mammographic findings of women treated with APBI using single-fraction IORT.

\section{Methods}

\subsection{Patient Selection}

Between December 2002-June 2004, women with Stage $0 / \mathrm{I}$ breast cancer were evaluated for an IRB-approved clinical trial with APBI. Inclusion criteria included age $\geq$ 40 with unifocal invasive ductal carcinoma (IDC) or ductal carcinoma in-situ (DCIS) measuring $\leq 2.5 \mathrm{~cm}$ on clinical or radiographic assessment. Exclusion criteria included IDC associated with an extensive intraductal component (EIC), invasive lobular carcinoma, tumor approximating or involving skin and/or pectoralis fascia, or involved axillary nodes. All patients underwent pre-operative mammography with ultrasound if clinically appropriate. Treatment options were presented to patients, including mastectomy, BCS with standard WBI, or APBI with IORT at the time of BCS. Patients underwent preoperative, contrast-enhanced breast magnetic resonance imaging (MRI) to confirm unifocal disease. Abnormal MRI findings were evaluated by US- or MRI-guided biopsy. Written informed consent was obtained from all patients prior to IORT by both the surgical and radiation oncology teams.

\subsection{Surgery and Intraoperative Radiotherapy}

Sentinel lymph node (SLN) biopsy, if indicated, was performed first followed by touch preparation or frozen section of excised SLN(s). SLN involvement precluded the use of IORT per protocol.

After excision of the lesion, specimen radiography was obtained to confirm excision with tumor-free margins. The breast tissue surrounding the cavity was then circumferentially freed from the skin anteriorly and from the pectoralis major muscle posteriorly for a distance of $5-10 \mathrm{~cm}$ from the edge of the cavity as described by Veronesi et al. [17,19]. A $3 \mathrm{~mm}$ lead plate was placed upon the pectoralis major muscle to limit the chest wall from exit radiation dose. The mobilized lumpectomy cavity margins were temporarily approximated to each other with Prolene sutures (Johnson and Johnson/Ethicon, Somerville, N.J.) over the lead plate. The target volume for irradiation was defined to include $1-3 \mathrm{~cm}$ of the lumpectomy cavity margins circumferentially.

A self-retaining retractor (Lone Star Medical Products, Inc. Stafford, TX) was used to retract skin and a $5-7.5$ $\mathrm{cm}$ diameter collimator was placed directly over the target volume. The collimator was then mounted to a Phil- lips 250 RT orthovoltage radiation unit. Seventeen Gy (surface dose) was delivered to the target volume in a single fraction over the course of $15-20$ minutes. At the completion of radiotherapy, the collimator and lead plate were removed, and the incision was closed over a small closed-suction drain (Jackson-Pratt ${ }^{\mathbb{B}}$, Cardinal Health, Inc, Dublin, Ohio) that remained in place for 3 - 10 days to minimize seroma formation. All patients received prophylactic antibiotics until drain removal.ase do not revise any of the current designations.

\subsection{Follow-Up Evaluation and Post-Operative Imaging}

Per protocol, treated patients were seen after surgery at 1 week, 1 month, 3 months, every 3 months for 2 years, then every 6 months.

If final pathology deemed margins $<2 \mathrm{~mm}$, the recommendation was to proceed with re-excision lumpectomy followed by WBI. Women who were found to have lymph node involvement on final pathology were advised to undergo completion axillary lymph node dissection (per standard recommendations at that time) as well as WBI. Adjuvant systemic therapy was left to the discretion of the medical oncologists.

Skin changes identified on physical examination, complications, and disease status were documented at each follow up visit. Fine needle aspiration (FNA), core needle biopsy, or excisional biopsy was performed for any clinical or radiologic abnormalities suspicious for recurrence or a new primary.

Patients underwent unilateral diagnostic mammography of the treated breast six months after lumpectomy and IORT. Bilateral mammograms were performed at one year, and then annually. Additional images were obtained as determined by our breast imagers. Three radiologists (DI, JA, SP) retrospectively reviewed and recorded mammographic findings, including breast tissue density, the presence or absence of masses or microcalcifications, and changes in the skin and irradiated tissue.

\section{Results}

Before you begin to format your paper, first write and save the content as a separate text file. Keep your text and graphic files separate until after the text has been formatted and styled. Do not use hard tabs, and limit use of hard returns to only one return at the end of a paragraph. Do not add any kind of pagination anywhere in the paper. Do not number text heads - the template will do that for you.

Finally, complete content and organizational editing before formatting. Please take note of the following items 
when proofreading spelling and grammar:

\subsection{Pre-Treatment Lesion Characteristics and Final Tumor Pathology}

During the study period 17 patients underwent IORT at the time of lumpectomy $(\mathrm{n}=11)$ or re-excision lumpectomy $(\mathrm{n}=6)$ (median age $=60$ years; range $=40-83$ ). At initial presentation, 14 patients $(82 \%)$ had lesions that were non-palpable while $3(18 \%)$ had palpable tumors. Pre-operative mammography demonstrated 16 of the 17 lesions (94\%), characterized as masses having irregular or spiculated margins with $(n=1)$ or without microcalcifications $(\mathrm{n}=13)$, or pleomorphic microcalcifications without an associated mass $(n=2)$. Fourteen patients underwent breast ultrasound for further characterization of palpable findings $(n=3)$ or for abnormalities seen on mammography $(\mathrm{n}=11)$. Pre-operative breast MRI in all 17 patients confirmed unifocal disease.

Eleven of the 17 patients $(65 \%)$ underwent primary lumpectomy and $6(35 \%)$ re-excision lumpectomy at the time of IORT. Fourteen of the 17 tumors (82\%) were IDC, 12 of which had associated DCIS. Three patients had DCIS. The median tumor size on final pathology was $13 \mathrm{~mm}$ (range $=1-24 \mathrm{~mm})$.

\subsection{Post-Treatment Follow up and Imaging}

At a median follow-up of 95 months (range $=86-104$ months), one patient had a local recurrence. While all patients were free of breast cancer distant metastasis, one patient died of colon cancer. All 17 patients had their first post-treatment mammogram 4 - 6 months after IORT. On the initial post-IORT mammogram, the lumpectomy/ IORT site showed increased density, as compared to the pre-treatment mammogram, in 11 patients $(65 \%)$, with 8 having density changes in a cylindrical-shaped pattern extending from the skin toward the chest wall corresponding to the target volume irradiated (Figure 1).

The remaining six patients $(35 \%)$ had architectural distortion in the area of the irradiated tissue. There was little edematous change in the breast tissue at the lumpectomy site.

Fifteen patients $(88 \%)$ had numerous scattered, punctate, benign-appearing calcifications that corresponded to the irradiated region and extended along the chest wall (Figure 2).

Some of these were fine, round calcifications while others were more coarse and dense. Despite the lack of skin irradiation, 13 patients (76\%) showed skin thickening near the skin incision, consistent with post-surgical healing. Skin away from the biopsy site was of normal thickness.

Long-term mammographic imaging was available for

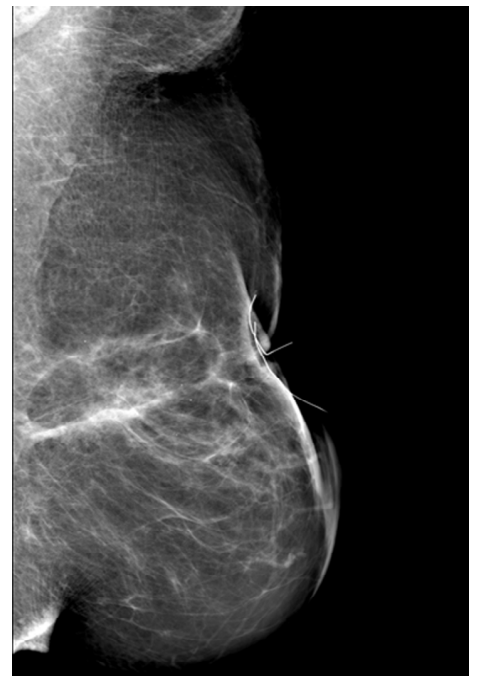

(a)

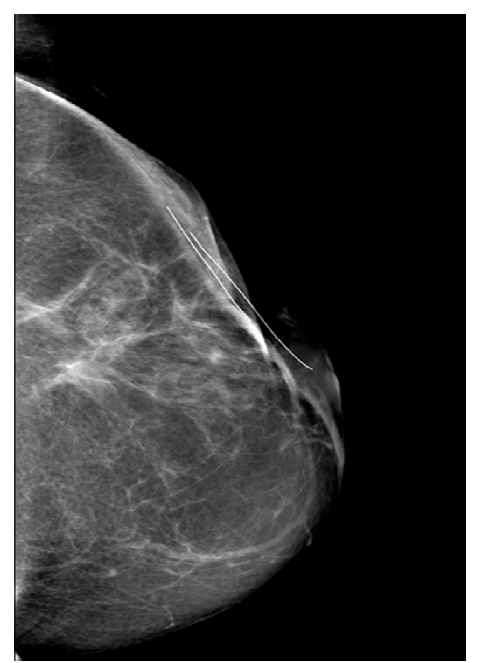

(b)

Figure 1. Unilateral mammogram 16 months post IORT in a 58 year old woman with high grade DCIS. (a) MLO view with focal cylindrical density extending from the scar to the chest wall. This density corresponds to the irradiated tissue; (b) CC view again demonstrating the asymmetric changes in the medial breast extending from the skin to the chest wall, the area treated with the single fraction intra-operative radiation.

15 patients at a median of 67 months (range 19 - 101) from the IORT treatment. In the 8 patients with cylindrical densities corresponding to the irradiated region, the area of increased density continued to decrease over time (Figure 3).

Architectural distortion was noted in all 15 patients and had stabilized over time. All patients had benignappearing microcalcifications that remained stable in number and character with longer follow up. No patient had a suspicious cluster of calcifications warranting biopsy. Focal skin thickening also persisted in a stable 


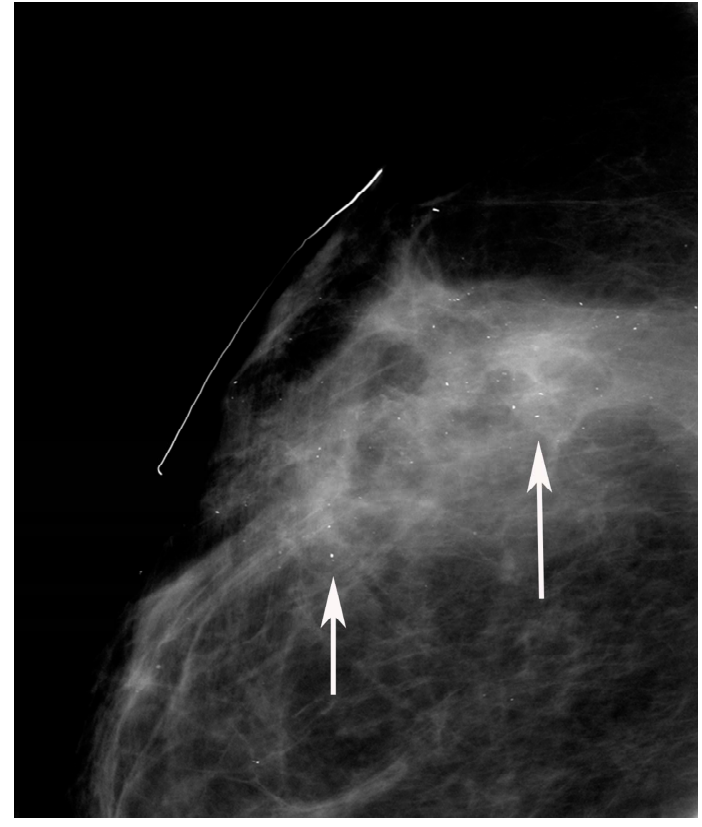

(a)

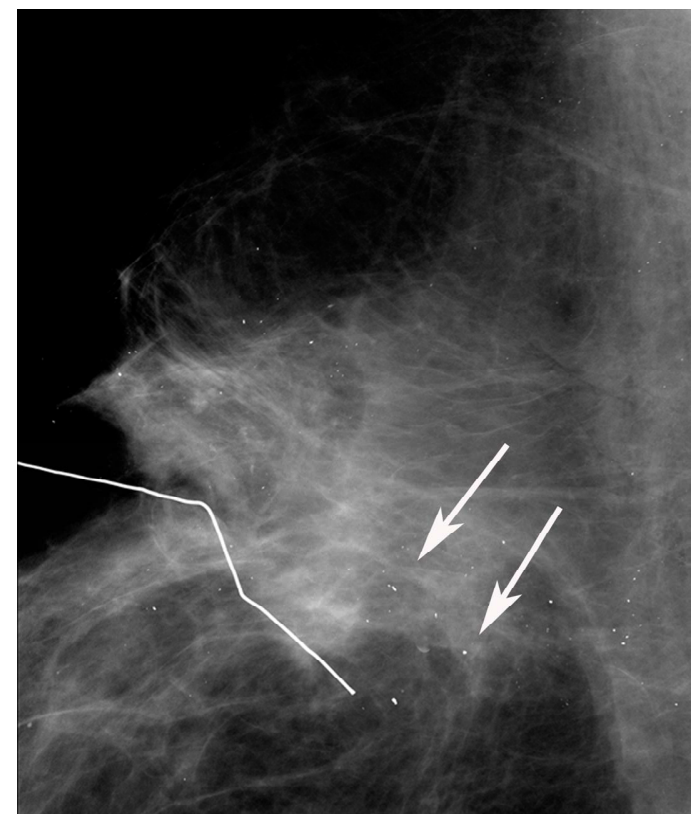

(b)

Figure 2. Magnification CC (a) and Lateral (b) mammogram in a woman treated 16 months earlier with IORT demonstrates numerous round, smooth, small calcifications (arrows) throughout most of the density that conforms to the single fraction intra-operative irradiated tissue and along the chest wall.

\section{fashion.}

Eight patients (47\%) had mammographic findings consistent with fat necrosis, zranging in size from $0.5-4 \mathrm{~cm}$. In patients who did not develop fat necrosis, most had heterogeneously dense breast tissue, while those who did

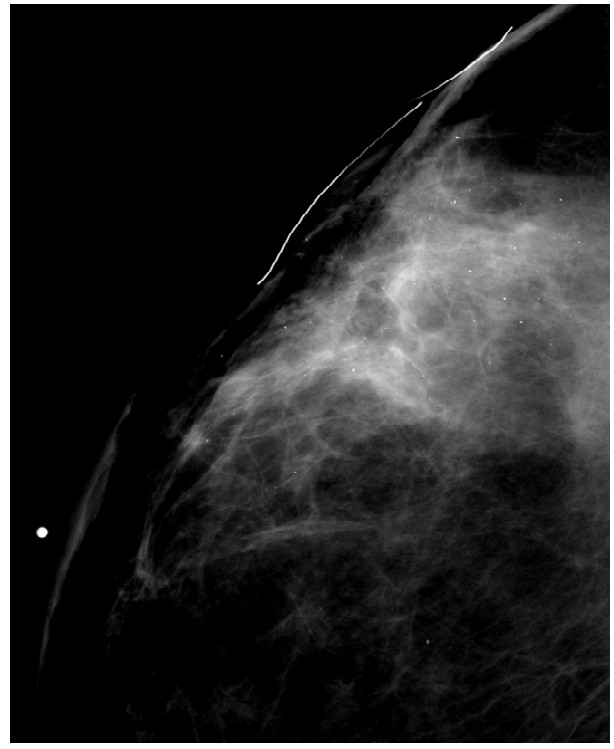

(a)

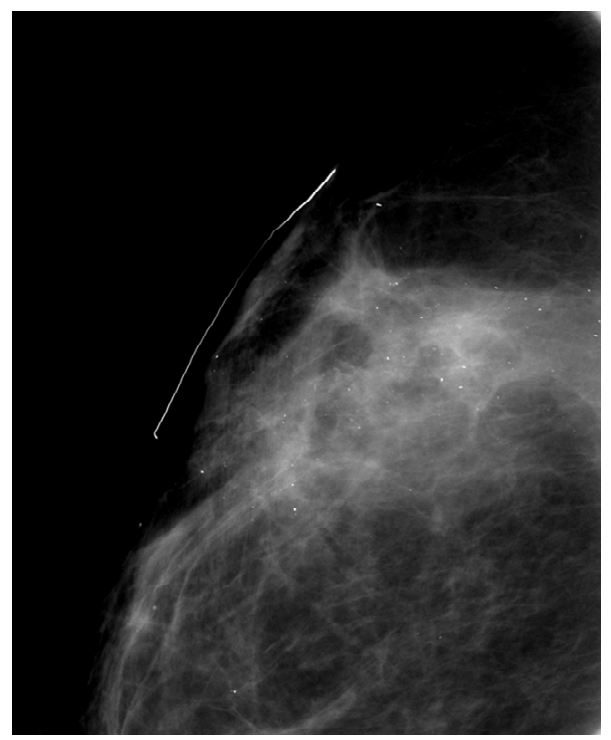

(b)

Figure 3. Magnification views at 10 months post-IORT treatment (a) and 16 months post-IORT (b). Focused density decreased over time while the calcifications remained the same.

develop fat necrosis mostly had scattered fibroglandular breast tissue.

\section{Discussion}

Breast conservation therapy alters the mammographic appearance of the treated breast. In one of the earliest papers discussing microcalcifications after BCS and WBI, Libshitz et al. characterized microcalcifications in the irradiated tissue in comparison to microcalcifications that indicated recurrent cancer [20]. Orel et al. further noted 
that benign calcifications in the irradiated field progressed over time from fine microcalcifications to dystrophic calcifications [21]. Dershaw et al. also described new faint, punctate calcifications that were clearly dissimilar from calcifications associated with breast carcinoma, which are usually irregular, branching, and/or pleomorphic in appearance [22]. These earlier descriptions of mammographic changes after WBI were important in defining post-treatment changes, such as skin thickening, increased tissue density, architectural distortion, increased trabecular thickening, edema, and/or microcalcifications, in contrast to changes associated with in-breast tumor recurrences (IBTR). After BCS and WBI, edema and increased tissue density typically resolve as the acute inflammation gives way to fibrosis and scarring, which are chronic tissue changes that occur as a result of radiation damage to the microvasculature. Microcalcifications typically form later and range from punctate calcifications to more coarse calcifications that have either radiolucent centers or a lipid cyst with curvilinear microcalcifications at its margin.

Mammographic changes after APBI using IORT, however, tend to be more focal in nature and differ in time course. In our patients, we found that skin thickening was localized to the surgical incision and was noted at the first 6-month post-treatment mammogram. The increased tissue density conformed to the focal area of irradiated tissue, developed early, and decreased over time. We did not identify significant fibrosis in these patients, even with long-term follow up. Benign calcifications appeared in $88 \%$ of our patients at the first mammogram and remained stable in number and appearance over time. Calcifications consistent with fat necrosis developed in $47 \%$, and were more common in patients with lower breast density.

Our findings are consistent with those reported in the literature. Series investigating IORT as a boost or as sole radiation treatment with either intraoperative electrons or low energy X-rays have reported similar post-treatment mammographic findings. Della Sala et al. and Carvalho et al. reported early changes after IORT using electrons that consisted of cutaneous thickening, architectural distortion, and benign calcifications which developed as early as 6 - 12 months after treatment and remained stable or even increased up to 24 months $[23,24]$. Wasser et al. found distinct, focal mammographic changes that appeared to remain stable with long-term follow up after IORT using $50 \mathrm{kV}$ with or without WBI [25]. Consistent with our findings, Kuzmiak et al. also found that mammographic changes varied according to breast density, with surgical scarring less apparent in those with lower breast density [26].

These distinct mammographic changes appear to be similar regardless of the APBI technique utilized. Lawenda et al. identified architectural distortion and calcifications in a series of patients treated on a dose escalation study using low-dose-rate interstitial brachytherapy with $192 \mathrm{Ir}$. The likelihood of having mammographic changes increased with dose escalation, with $36.8 \%, 40 \%$, and $50 \%$ having focal mammographic findings in those treated with 50, 55, and $60 \mathrm{~Gy}$, respectively [27]. Esserman et al. reported imaging findings in patients treated with APBI using interstitial or intracavitary brachytherapy [28]. Fortyone of 43 patients $(95 \%)$ had focal increased density, and 8 patients (19\%) had calcifications typical for fat necrosis appearing at 12 months of follow up. Ahmed et al. reported focal findings consisting of fat necrosis, seroma formation, and architectural distortion in patients treated with MammoSite balloon brachytherapy which peaked at 21 months of follow up [29].

The findings with interstitial or intracavitary brachytherapy, which often delivers high or low-dose radiotherapy to the lumpectomy cavity twice daily over $4-5$ days, suggest that radiographic evidence of fat necrosis may vary from approximately $3 \%$ to up to $19 \%-20 \%$ [27-30]. With single fraction IORT, the radiographic description of fat necrosis is higher, ranging from $20 \%$ $57 \%$ supporting the concern that breast tissue damage is, indeed, likely related to the dose delivered per fraction $[23,24,31]$. Interestingly, the appearance of microcalcifications in our cohort occurred earlier and more frequently than what is typically seen with standard fractionated breast radiotherapy. However, they remained stable in number, size, and character with long-term follow up. In contrast, patients who were treated with APBI using high-dose-rate brachytherapy appear to have a lower rate of post-treatment microcalcifications initially, but these seem to increase over time [32]. Interestingly, most of these radiographic changes consistent with fat necrosis continue to be asymptomatic. Longer follow-up will be necessary to determine how these findings evolve.

This study is limited by its retrospective nature, the small sample size, and the fact that the breast radiologists were not blinded. Our results therefore need to be confirmed in a larger series of patients treated with APBI using IORT. The recently reported TARGIT trial (ref), reported equivalent results of IORT compared to WBI after BCS, however, no post-treatment imaging data are available at this time [13].

Our findings confirm that mammographic changes after IORT, such as increased tissue density, architectural distortion, and benign-appearing calcifications, appear early in the irradiated tissue yet tend to decrease or remain stable over time. These findings can help guide 
physicians in radiographic follow up of patients treated with APBI.

\section{REFERENCES}

[1] J. A. Jacobson, D. N. Danforth, K. H. Cowan, T. d'Angelo, S. M. Steinberg, L. Pierce, et al., "Ten-Year Results of a Comparison of Conservation with Mastectomy in the Treatment of Stage I and II Breast Cancer," New England Journal of Medicine, Vol. 332, No. 14, 1995, pp. 907-911. doi:10.1056/NEJM199504063321402

[2] B. Fisher, S. Anderson, J. Bryant, R. G. Margolese, M. Deutsch, E. R. Fisher, et al., "Twenty-Year Follow-Up of a Randomized Trial Comparing Total Mastectomy, Lumpectomy, and Lumpectomy Plus Irradiation for the Treatment of Invasive Breast Cancer," New England Journal of Medicine, Vol. 347, No. 16, 2002, pp. 1233-1241. doi:10.1056/NEJMoa022152

[3] R. Arriagada, M. G. Le, F. Rochard and G. Contesso, "Conservative Treatment versus Mastectomy in Early Breast Cancer: Patterns of Failure with 15 Years of Follow-Up Data. Institut Gustave-Roussy Breast Cancer Group," Journal of Clinical Oncology, Vol. 14, No. 5, 1996, pp. 1558-1564.

[4] M. Blichert-Toft, C. Rose, J. A. Andersen, M. Overgaard, C. K. Axelsson, K. W. Andersen, et al., "Danish Randomized Trial Comparing Breast Conservation Therapy with Mastectomy: Six Years of Life-Table Analysis. Danish Breast Cancer Cooperative Group," Journal of the National Cancer Institute Monographs, Vol. 11, 1992, pp. 19-25.

[5] J. A. van Dongen, A. C. Voogd, I. S. Fentiman, C. Legrand, R. J. Sylvester, D. Tong, et al., "Long-Term Results of a Randomized Trial Comparing Breast-Conserving Therapy with Mastectomy: European Organization for Research and Treatment of Cancer 10801 Trial," Journal of the National Cancer Institute, Vol. 92, No. 14, 2000, pp. 1143-1150. doi:10.1093/jnci/92.14.1143

[6] U. Veronesi, N. Cascinelli, L. Mariani, M. Greco, R. Saccozzi, A. Luini, et al., "Twenty-Year Follow-Up of a Randomized Study Comparing Breast-Conserving Surgery with Radical Mastectomy for Early Breast Cancer," New England Journal of Medicine, Vol. 347, 2002, pp. 1227-1232. doi:10.1056/NEJMoa020989

[7] M. Morrow, "Rational Local Therapy for Breast Cancer," New England Journal of Medicine, Vol. 347, 2002, pp. 1270-1271. doi:10.1056/NEJMe020112

[8] H. M. Kuerer, T. B. Julian, E. A. Strom, H. K. Lyerly, A. E. Giuliano, E. P. Mamounas, et al., "Accelerated Partial Breast Irradiation after Conservative Surgery for Breast Cancer," Annals of Surgery, Vol. 239, No. 3, 2004, pp. 338-351. doi:10.1097/01.sla.0000114219.71899.13

[9] M. Keisch, F. Vicini, R. Kuske, M. Hebert, J. White, C. Quiet, et al., "Two-Year Outcome with the MammoSite Breast Brachytherapy Applicator: Factors Associated with Optimal Cosmetic Results When Performing Partial Breast Irradiation," International Journal of Radiation Oncology Biology Physics, Vol. 57, No. 2, 2003, p. pS315.

\section{doi:10.1016/S0360-3016(03)01186-6}

[10] F. A. Vicini, V. Remouchamps, M. Wallace, M. Sharpe, J. Fayad, L. Tyburski, et al., "Ongoing Clinical Experience Utilizing 3D Conformal External Beam Radiotherapy to Deliver Partial-Breast Irradiation in Patients with EarlyStage Breast Cancer Treated with Breast-Conserving Therapy," International Journal of Radiation Oncology Biology Physics, Vol. 57, No. 5, 2003, pp. 1247-1253. doi:10.1016/S0360-3016(03)01573-6

[11] F. M. Dirbas, S. S. Jeffrey and D. R. Goffinet, "The Evolution of Accelerated Partial Breast Irradiation as a Potential Treatment Option for Women with Newly Diagnosed Breast Cancer Considering Breast Conservation," Cancer Biotherapy and Radiopharmaceuticals, Vol. 19, No. 6, 2004, pp. 673-705. doi:10.1089/cbr.2004.19.673

[12] U. Veronesi, R. Orecchia, A. Luini, V. Galimberti, G. Gatti, M. Intra, et al., "Full-Dose Intraoperative Radiotherapy with Electrons during Breast-Conserving Surgery: Experience with 590 Cases," Annals of Surgery, Vol. 242, No. 1, 2005, pp. 101-106. doi:10.1097/01.sla.0000167927.82353.bc

[13] J. S. Vaidya, D. J. Joseph, J. S. Tobias, M. Bulsara, F. Wenz, C. Saunders, et al., "Targeted Intraoperative Radiotherapy versus Whole Breast Radiotherapy for Breast Cancer (TARGIT-A trial): An International Prospective, Randomised, Non-Inferiority Phase 3 Trial," The Lancet, Vol. 376, No. 9735, 2010, pp. 91-102. doi:10.1016/S0140-6736(10)60837-9

[14] C. Rose and A. Recht, "Accelerated Partial-Breast Irradiation (APBI): Let's Give It a Good Test," International Journal of Radiation Oncology Biology Physics, Vol. 57, No. 5, 2003, pp. 1217-1218. doi:10.1016/S0360-3016(03)01572-4

[15] L. Solin, B. Fowble, R. Troupin and R. Goodman, "Biopsy Results of New Calcifications in the Postirradiated Breast," Cancer, Vol. 63, No. 10, 1989, pp. 1956-1961. doi:10.1002/1097-0142(19890515)63:10<1956::AID-CN CR2820631015>3.0.CO;2-N

[16] K. Harris, M. Costa-Greco, A. Baratz, C. Britton, Z. Ilkhanipour and M. Ganott, "The Mammographic Features of the Postlumpectomy, Postirradiation Breast," Radiographics, Vol. 9, 1989, pp. 253-268.

[17] U. Veronesi, G. Gatti, A. Luini, M. Intra, R. Orecchia, P. Borgen, et al., "Intraoperative Radiation Therapy for Breast Cancer: Technical Notes," The Breast Journal, Vol. 9, No. 2, 2003, pp. 106-112. doi:10.1046/j.1524-4741.2003.09208.x

[18] H. Bartelink, "Commentary on the Paper 'A Preliminary Report of Intraoperative Radiotherapy (IORT) in Limited-Stage Breast Cancers That Are Conservatively Treated'-A Critical Review of an Innovative Approach," European Journal of Cancer, Vol. 37, No. 17, 2001, pp. 2143-2146. doi:10.1016/S0959-8049(01)00284-2

[19] M. Intra, G. Gatti, A. Luini, V. Galimberti, P. Veronesi, S. Zurrida, et al., "Surgical Technique of Intraoperative Radiotherapy in Conservative Treatment of Limited-Stage Breast Cancer," Archives of Surgery, Vol. 137, No. 6, 
2002, pp. 737-740. doi:10.1001/archsurg.137.6.737

[20] H. I. Libshitz, E. D. Montague and D. D. Paulus, "Calcifications and the Therapeutically Irradiated Breast," American Journal of Roentgenology, Vol. 128, No. 6, 1977, pp. 1021-1025.

[21] S. G. Orel, R. H. Troupin, E. A. Patterson and B. L. Fowble, "Breast Cancer Recurrence after Lumpectomy and Irradiation: Role of Mammography in Detection," Radiology, Vol. 183, No. 1, 1992, pp. 201-206.

[22] D. D. Dershaw, "Evaluation of the Breast Undergoing Lumpectomy and Radiation Therapy," Radiological Clinics of North America, Vol. 33, No. 6, 1995, pp. 11471160.

[23] B. Carvalho, A. Frasson, M. Santos and N. de Barros, "Mammography Findings Following Electron Intraoperative Radiotherapy or External Radiotherapy for Breast Cancer Treatment," European Journal of Radiology, Vol. 79, No. 2, 2010, pp. e7-e10.

[24] S. W. Della Sala, M. Pellegrini, D. Bernardi, F. Franzoso, M. Valentini, S. Di Michele, et al., "Mammographic and Ultrasonographic Comparison between Intraoperative Radiotherapy (IORT) and Conventional External Radiotherapy (RT) in Limited-Stage Breast Cancer, Conservatively Treated," European Journal of Radiology, Vol. 59, No. 2, 2006, pp. 222-230. doi:10.1016/i.ejrad.2006.03.003

[25] K. Wasser, M. Ruch, J. Brade, C. Schoeber, U. KrausTiefenbacher, A. Schnitzer, et al., "Do Structural Changes in the Tumour Bed after Intraoperative Radiotherapy (IORT) of Breast Cancer Complicate the Evaluation of Mammograms in a Long-Term Follow-Up?" European Journal of Radiology, Vol. 81, No. 3, 2011, pp. e255e259.

[26] C. M. Kuzmiak, D. Zeng, E. Cole and E. D. Pisano, "Mammographic Findings of Partial Breast Irradiation," Academic Radiology, Vol. 16, No. 7, 2009, pp. 819-825. doi:10.1016/j.acra.2009.01.021

[27] B. D. Lawenda, A. G. Taghian, L. A. Kachnic, H. Hamdi, B. L. Smith, M. A. Gadd, et al., "Dose-Volume Analysis of Radiotherapy for T1N0 Invasive Breast Cancer Treated by Local Excision and Partial Breast Irradiation by LowDose-Rate Interstitial Implant," International Journal of Radiation Oncology Biology Physics, Vol. 56, No. 3, 2003, pp. 671-680. doi:10.1016/S0360-3016(03)00071-3

[28] L. E. Esserman, M. d'Almeida, E. C. Gombos and M. Keisch, "Imaging Findings after Breast Brachytherapy," American Journal Roentgenology, Vol. 187, No. 1, 2006, pp. 57-64. doi:10.2214/AJR.05.0318

[29] H. M. Ahmed, P. J. DiPiro, P. M. Devlin, M. L. Nguyen and J. R. Bellon, "Mammographic Appearance Following Accelerated Partial Breast Irradiation by Using MammoSite Brachytherapy," Radiology, Vol. 255, No. 2, 2010, pp. 362-368. doi:10.1148/radiol.10091625

[30] D. E. Wazer, D. Lowther, T. Boyle, K. Ulin, A. Neuschatz, R. Ruthazer, et al., "Clinically Evident Fat Necrosis in Women Treated with High-Dose-Rate Brachytherapy Alone for Early-Stage Breast Cancer," International Journal of Radiation Oncology Biology Physics, Vol. 50, No. 1, 2001, pp. 107-111. doi:10.1016/S0360-3016(00)01541-8

[31] M. Ruch, J. Brade, C. Schoeber, U. Kraus-Tiefenbacher, A. Schnitzer, D. Engel, et al., "Long-Term Follow-Up Findings in Mammography and Ultrasound after Intraoperative Radiotherapy (IORT) for Breast Cancer," The Breast, Vol. 18, No. 5, 2009, pp. 327-334. doi:10.1016/j.breast.2009.09.010

[32] P. R. Benitez, P. Y. Chen, F. A. Vicini, M. Wallace, L. Kestin, G. Edmundson, et al., "Partial Breast Irradiation in Breast Conserving Therapy by Way of Interstitial Brachytherapy," American Journal of Surgery, Vol. 188, No. 4, 2004, pp. 355-364. doi:10.1016/j.amjsurg.2004.06.027 